\title{
Vulgarizing Post Exposure Prophylaxis to Complete the Comprehensive Prevention Package Is Vital In Reducing the Incidence of HIV/AIDS
}

\section{Gordon Kunbuma Tachang ${ }^{1 *}$ and Henry Dilonga Meriki}

${ }^{1}$ Harvard University School of Public Health, 677 Huntington Ave., Boston, MA, 02115, USA

${ }^{2}$ Buea Regional Hospital, Southwest Region, Cameroon

\begin{abstract}
Advancements in the fight against HIVIAIDS could be achieved by thinking differently, breaking paradigms and forging together in a shared vision of tackling one of the toughest challenges of mankind. Vulgarization of nonoccupational post exposure prophylaxis especially in low-income settings which bear the brunt of HIVIAIDS, could complement the comprehensive preventive strategies available to break the transmission chain and reduce the incidence of HIVIAIDS.
\end{abstract}

\section{Keywords: HIV/AIDS; PEP; Low-income settings}

As far as the HIV/AIDS pandemic is concerned, while some middle income countries are making remarkable progress, others are falling back. Besides HIV like TB is becoming less generalized and more concentrated, in certain areas and within key populations within a country. Thus a diversified and differential approach is required to achieve control and useful efforts should aim at pinpointing the geographic zones at sub national levels having the highest disease burden [1]. Once these zones are identified, and the most common mode of transmission ascertained, then a comprehensive prevention package can be put in place. This package should include non-occupational post exposure prophylaxis if the sexual mode of transmission is common in this locality.

Preventing an infection by the use of medicines shortly after exposure to a germ is called post-exposure prophylaxis (PEP). In the case of HIV/AIDS, the use of anti -retroviral therapy (ART) $24-72$ hours after potential exposure to the virus either occupationally or non -occupationally is termed PEP. The therapeutic basis for the use of PEP in humans is inspired by the experiments that were conducted in monkeys using anti- simian immunodefiency virus (SIV) drugs [2]. These drugs administered shortly after monkeys were exposed to the simian immunodefiency virus (SIV) greatly reduced their risk of becoming infected with the virus. These animal studies proved that PEP is most effective if the drugs are taken within maximum of 72 hours after exposure and for a period of at least 4 consecutive weeks. These medications prevent HIV from making copies of itself and spreading throughout the body.

Exposure to the HIV virus could be (1) occupational PEP (sometimes called "oPEP"), taken when someone working in a healthcare setting is potentially exposed to material infected with HIV, and (2) non-occupational PEP (sometimes called "nPEP"), taken when someone is potentially exposed to HIV outside the workplace [3] (e.g., from sexual assault, or during episodes of unprotected sex or needlesharing injection drug use). The use of post exposure prophylaxis presupposes that the person who was exposed to HIV is HIVnegative; thus, a negative result of a baseline enzyme-linked immune sorbent assay (ELISA) for antibodies to HIV should be documented concomitantly with the assessment for post exposure prophylaxis.

The nature of the exposure determines the per-contact risk of HIV transmission from the sexual contact. The estimated risks are 1 to $30 \%$ with receptive anal intercourse, 0.1 to $10.0 \%$ with insertive anal intercourse and receptive vaginal intercourse, and 0.1 to $1.0 \%$ with insertive vaginal intercourse [4]. Splashes of infectious material to mucous membranes (e.g., conjunctivae or oral mucosa) or broken skin also may transmit HIV infection (estimated risk per exposure [5], $0.09 \%$ [ $95 \%$ CI, 0.006 to 0.5$]$ ]). As compared with other forms of intercourse, oral intercourse is considered to pose a lower risk of HIV transmission, although good risk estimates are lacking, and there are case reports of HIV infections in persons in whom the only reported risk factor was oral intercourse [6].

Testing to assess the HIV viral load, in the absence of signs or symptoms that are consistent with primary HIV infection, is currently not routinely recommended because of concerns about false positive results and cost [7], although a qualitative nucleic acid amplification test has been approved by the Food and Drug Administration for this purpose. The early initiation of antiretroviral therapy reduced rates of sexual transmission of HIV-1 and clinical events, indicating both personal and public health benefits from such therapy. Antiretroviral therapy 1-that reduces viral replication could limit the transmission of human immunodeficiency virus type 1 (HIV-1) in serodiscordant couples [8]. Antiretroviral pre-exposure prophylaxis (PrEP) reduces the incidence of acquisition of human immunodeficiency virus type 1 (HIV-1) in a man who has sex with men and is a promising approach for preventing HIV-1 in heterosexual populations [9].

In most high-income countries, including Canada, PEP is freely available after a work-related exposure such as a needle-stick injury among health care workers [10]. However, the use of PEP is not generally subsidized for accidental exposure because of consensual sex in Canada. Still, PEP combined with intensive counseling about risk behavior may be a useful way of helping to limit the spread of HIV among sexually active people, who are at high risk for HIV infection, particularly men who have sex with men (MSM). This would surely reduce the incidence of the pandemic. Within the health sector, PEP

*Corresponding author: Gordon Kunbuma Tachang, Harvard University School of Public Health, 677 Huntington Ave., Boston, MA, 02115, USA, Tel: (617) 459 2242; E-mail: gkt402@mail.harvard.edu

Received June 15, 2014; Accepted August 20, 2015; Published August 31, 2015

Citation: Tachang GK, Meriki HD (2015) Vulgarizing Post Exposure Prophylaxis to Complete the Comprehensive Prevention Package Is Vital In Reducing the Incidence of HIVIAIDS. J AIDS Clin Res 6: 496. doi:10.4172/2155-6113.1000496

Copyright: (c) 2015 Tachang GK, et al. This is an open-access article distributed under the terms of the Creative Commons Attribution License, which permits unrestricted use, distribution, and reproduction in any medium, provided the original author and source are credited. 
Citation: Tachang GK, Meriki HD (2015) Vulgarizing Post Exposure Prophylaxis to Complete the Comprehensive Prevention Package Is Vital In Reducing the Incidence of HIVIAIDS. J AIDS Clin Res 6: 496. doi:10.4172/2155-6113.1000496

should be provided as part of a comprehensive universal precautions package that reduces staff exposure to infectious hazards at work.

In many low income settings like in some developed countries, PEP is reserved only for work related or occupational contamination only. No immediate action is taken against non-occupational PEP. It is worth noting here that this is a misnomer since it is cheaper and smarter to treat a patient with antiretroviral for duration of just one month than for a whole lifetime. This is particularly relevant in this era of reduced availability of funds. The case in point here is the non-occupational PEP which has an $80 \%$ chance of preventing HIV especially if the one month treatment of antiretroviral is taken very faithfully. Counseling and PEP must go hand in hand for non-occupational PEP to be successful.

Although in resource limited settings (RLS), antiretroviral therapy (ART) care and treatment is largely supported by external funding (PEPFAR, Global Funds), some governments in the resource limited settings have been unable to contribute their token quota towards the purchase of anti-retroviral for their HIV positive patients. Thus the introduction of non-PEP to complete the combination of more comprehensive preventive strategies [9] will surely reduce the incidence of the HIV in these low income countries. Information regarding the exact protocol to adopt by the general population in the event of a potential exposure to the HIV is not yet well vulgarized within most communities in low income settings despite the fact that the benefits of PEP have been published and are known to care providers and policy audiences. The erroneous or one sided view by some health officials in low income settings that vulgarizing PEP might rekindle behavioral complaisance to the comprehensive prevention strategies for HIV/AIDS is only partially justified if at all. This may be hurting to the prevention campaigns because in the event of unsafe casual sex under the influence of alcohol or episodes of broken condoms, the subject is allowed to him/herself with the likelihood of contracting the virus. Care must be taken to point out that the PEP must not replace the comprehensive preventive strategies for HIV/AIDS geared at avoiding HIV exposure (e.g., sexual abstinence, sex only in a mutually monogamous relationship with a non-infected partner, consistent and correct condom use, abstinence from injection-drug use, and consistent use of sterile equipment by those unable to cease injection-drug use).

Efforts to promote adherence to post exposure prophylaxis and referrals for counseling regarding risk reduction, as well as mental health, substance abuse, and domestic violence services, as appropriate, should be considered to be an integral part of programs for patients who receive post exposure prophylaxis. Moreover, the common side effects from PEP medications are nausea, headaches, fatigue, vomiting, diarrhea and generally not feeling well. These side effects which are generally quite discomforting can serve as a deterrent to unsafe sexual habits in future. In another study where men who were exposed to HIV were given counseling and PEP, 77\% of participants reported a decline in unprotected sex after counseling [11].

Thus after a potentially high risk exposure, especially with high risk groups [such as MSM, Sex workers, rape survivor] or in the event of condom breakage or unprotected casual sex, non-occupational PEP must be administered if subject tests negative for HIV. The new WHO guidelines for putting all people infected with HIV on ART as soon as diagnosed (Test and Treat) to reduce incidence would surely have fewer infected persons to handle, thanks to the non-occupational PEP. The TasP (treatment as prevention) paradigm is well-accepted internationally, and increased attention to the use of PrEP (pre-exposure prophylaxis) should be widely used among high risk populations.
Although medical treatment after non-occupational HIV exposure is less cost-effective than preventing HIV infection by avoiding exposure, non-occupational PEP earns its ultimate relevance as the last resort only when the comprehensive preventive strategies fail. In most low income settings, this very vital information is unavailable to the general population as only the occupational PEP has been publicized. New strategies on enticing the general population and especially the sexually active age group to undergo screening for the HIV/AIDS must be envisaged. This is particularly relevant as the most dangerous persons in any community are individuals who have never known their serological status.

Vulgarizing the use of non-occupational post exposure prophylaxis especially in low income settings strengthens and completes the comprehensive preventive strategies needed to break the transmission chain and reduce the incidence of HIV/AIDS to zero. The future of HIV/AIDS shall be forged by thinking differently, breaking paradigms and forging together in a shared vision of tackling one of the toughest challenges before mankind-the HIV/AIDS pandemic. No one country can do it alone: achieving an AIDS-free generation is a shared responsibility [12].

\section{References}

1. WHO, UNAIDS (2013) Global Update on HIV Treatment 2013: Results, Impact and Opportunities. WHO Report in partnership with UNICEF and UNAIDS.

2. Van Rompay KKA, Marthas ML, Ramos RA, Mandell, CP, McGowan EK, et al. (1992) Simian immunodeficiency virus (SIV) infection of infant rhesus macaques as a model to test antiretroviral drug prophylaxis and therapy: oral 3'-azido-3'-deoxythymidine prevents SIV infection. Antimicrob. Agents Chemother 36: 2381-2386.

3. Centre for Disease Control and prevention. About HIV PEP.

4. Cohen MS, Chen YQ, McCauley M, Gamble T, Hosseinipour MC, et al. (2011) Prevention of HIV-1 infection with early antiretroviral therapy. N Engl J Med 365: 493-505.

5. Attia S, Egger M, Müller M, Zwahlen M, Low N (2009) Sexual transmission of HIV according to viral load and antiretroviral therapy: systematic review and meta-analysis. AIDS 23: 1397-1404

6. Landovitz RJ, Currier JS (2009) Clinical practice. Postexposure prophylaxis for HIV infection. N Engl J Med 361: 1768-1775.

7. Donnell D, Baeten JM, Kiarie J, Thomas KK, Stevens W, et al. (2010) Heterosexual HIV-1 transmission after initiation of antiretroviral therapy: a prospective cohort analysis. Lancet 375: 2092-2098.

8. Cohen MS, Chen YQ, McCauley M, Gamble T, Hosseinipour MC, et al. (2011) Prevention of HIV-1 infection with early antiretroviral therapy. N Engl J Med 365: 493-505

9. Sean R Hosein (2012) Unexpected Effects of PEP in Amsterdam Canadian AIDS Treatment Information Exchange.

10. CATIE Canadian source for HIV and hepatitis C information (2012) Treatment update 188.

11. Martin JN, Roland ME, Neilands TB, Krone MR, Bamberger JD, et al. (2004) Use of postexposure prophylaxis against HIV infection following sexual exposure does not lead to increases in high-risk behavior. AIDS 18: 787-792.

12. PEPFAR (2014) Tenth Annual Report to Congress on PEPFAR (2014). 